\title{
An HGF-dependent positive feedback loop between bladder cancer cells and fibroblasts mediates lymphangiogenesis and lymphatic metastasis
}

Changhao Chen ( $\square$ chenchh53@mail.sysu.edu.cn )

Sun Yat-sen Memorial Hospital https://orcid.org/0000-0002-9924-9054

\section{Yuting Li}

Sun Yat-sen Memorial Hospital

\section{Yuming Luo}

Guangdong Provincial People's Hospital

\section{Hanhao Zheng}

Sun Yat-sen Memorial Hospital

\section{Yan Lin}

Sun Yat-sen Memorial Hospital

\section{Mingjie An}

Sun Yat-sen Memorial Hospital

\section{Yao Kong}

Guangdong Provincial People's Hospital

\section{Yue Zhao}

Sun Yat-sen University First Affiliated Hospital

\section{Yina Yin}

Sun Yat-sen Memorial Hospital

\section{Le Ai}

Sun Yat-sen Memorial Hospital

\section{Yiyao Ya}

Sun Yat-sen Memorial Hospital

Jian Huang

Sun Yat-sen Memorial Hospital

\section{Zhihua Li}

Sun Yat-sen Memorial Hospital

\section{Article}

Keywords: Cancer-associated fibroblasts, Extracellular vesicle, Long noncoding RNA, Lymphangiogenesis, Bladder cancer, HGF 
Posted Date: December 9th, 2021

DOI: https://doi.org/10.21203/rs.3.rs-1104668/v1

License: (c) (i) This work is licensed under a Creative Commons Attribution 4.0 International License. Read Full License 


\section{Abstract}

Cancer-associated fibroblasts (CAFs) are essential etiologic actors in promoting tumor progression via extensive reciprocal interactions with cancer cells. Yet, the biological role and regulatory mechanism of CAFs phenotype underlying lymph node (LN) metastasis of bladder cancer (BCa) remain unclear. Here, we report that BCa cell-secreted extracellular vesicles (EVs) played an important role in the CAF-enriched microenvironment, which correlated with BCa lymphangiogenesis and LN metastasis. RNA sequencing identified an EV-associated long noncoding RNA, LINC00665, which acted as a crucial mediator of CAF infiltration in BCa. LINC00665 mediated EV release from BCa cells to endow fibroblasts with the CAF phenotype, which reciprocally induced LINC00665 upregulation to form a RAB27B-HGF-c-Myc positive feedback loop, facilitating BCa lymphangiogenesis and LN metastasis. Importantly, we demonstrate that Cabozantinib significantly suppressed LINC00665-mediated BCa LN metastasis in an orthotopic xenograft model. Our study highlights a molecular mechanism by which LINC00665 induces a RAB27BHGF-c-Myc positive feedback loop between cancer cells and fibroblasts to sustain BCa LN metastasis, and represents LINC00665 as a potential therapeutic target in BCa LN metastasis.

\section{Introduction}

Bladder cancer $(\mathrm{BCa})$ is one of the most commonly diagnosed urinary malignancies, with an estimated yearly 550,000 new cases worldwide. Based on muscle infiltration, BCa is divided into non-muscleinvasive bladder cancer (NMIBC) and muscle-invasive bladder cancer (MIBC) ${ }^{1,2}$. Intensive research has demonstrated that MIBC is accompanied by a particularly tumorigenic microenvironment to acquire a higher incidence of lymph node (LN) metastasis than NMIBC, leading to the poor prognosis ${ }^{3,4}$. Cisplatinbased neoadjuvant therapy combined with radical cystectomy is acknowledged as the standard treatment for patients with advanced $\mathrm{BCa}$, which has limited benefit in improving the 5-year survival rate of patients with LN metastasis, showing the great challenge in developing new therapeutic strategies for $\mathrm{LN}$ metastatic $\mathrm{BCa}^{5}$. Cabozantinib, a small-molecular inhibitor specifically targeting the HGF-Met signaling pathway, has been approved by the US Food and Drug Administration for its striking clinical efficacy in suppressing tumor angiogenesis ${ }^{6,7}$. Nevertheless, the clinical application potential of Cabozantinib in LN metastatic BCa remains unclear.

Lymphangiogenesis refers to the sprouting of lymphatic vasculature in the microenvironment, which enlarges the lymphatic draining system for disseminating cancer cells ${ }^{8,9}$. We and others have demonstrated that regulatory signals from the tumor microenvironment (TME) are essential for lymphangiogenesis and predict increased LN metastasis and poor prognosis of patients ${ }^{10-12}$. However, the predominant mediators in TME that trigger lymphangiogenesis are largely unknown. Cancerassociated fibroblasts (CAFs) are the major stromal cell component in the TME that generate various cytokines to shape the desmoplastic microenvironment, resulting in tumor therapeutic resistance ${ }^{13,14}$. Moreover, the molecular signal from CAFs is efficiently transferred to cancer cells to regulate cancer cell epithelial-mesenchymal transition and stemness and to enable tumor dissemination ${ }^{15,16}$. Therefore, 
elucidating the biological role and regulatory mechanisms of CAFs in mediating $\mathrm{BCa}$ lymphangiogenesis and LN metastasis is of great significance.

The precise interaction profile between tumor cells and CAFs are under intensive exploration, where the extracellular vesicles (EVs) serving as crucial cargo carriers of diverse pro-tumor molecules to mediate intercellular communication have received close attention ${ }^{17,18}$. Tumor cell-derived EVs specifically modify the stromal cellular phenotype and reshape the TME to form a premetastatic niche and support tumor metastasis ${ }^{19}$. Conversely, EVs released from CAFs are widely internalized by tumor cells and contribute to the activation of aggressive tumor biology ${ }^{20}$. Inhibiting the EV-mediated crosstalk between tumor cells and CAFs has a substantial biological effect in suppressing tumor metastasis and therapy resistance ${ }^{21}$. However, the molecular mechanism of EVs in regulating the trafficking among tumor cells and CAFs to induce BCa lymphangiogenesis is not well-elucidated.

Long noncoding RNAs (IncRNAs) act as vital regulators in activating the signaling cascade in the TME ${ }^{22}$. Yet, the essential EV-associated IncRNAs involved in mediating the CAF phenotype are largely unexplored. In the present study, we identified an EV-associated IncRNA, LINC00665, that is overexpressed in LN metastatic BCa. LINC00665 activated RAB27B transcription and promoted EV production by BCa cells to trigger fibroblast transition to CAFs, which in turn, upregulated LINC00665 expression to form a RAB27BHGF-c-Myc positive feedback loop between BCa cells and fibroblasts, ultimately facilitating BCa lymphangiogenesis and LN metastasis. Importantly, cabozantinib treatment in an orthotopic xenograft model markedly inhibited LINC00665-induced BCa LN metastasis. Our study highlights a novel mechanism by which LINC00665-induced EV-mediated RAB27B-HGF-c-Myc positive feedback between $\mathrm{BCa}$ cells and fibroblasts contributes to lymphangiogenesis and LN metastasis, indicating that targeting LINC00665 may serve as a synergistic treatment for patients with LN metastatic BCa.

\section{Results}

\section{LINC00665 is associated with CAF-induced LN metastasis of BCa.}

Lymphangiogenesis contributes to the uncontrolled formation of a dysfunctional lymphatic network with incomplete basement membranes, which is considered the rate-limiting step in the metastasis of malignant cells through $\mathrm{LNs}^{23}$. Therefore, we collected BCa tissues from 228 cases at Sun Yat-Sen Memorial Hospital and subjected them to immunohistochemistry $(\mathrm{IHC})$ analysis for lymphangiogenesis. The results showed that $L N$ metastatic $B C a$ tissues were presented with higher microlymphatic vessel density (MLD) than those without LN metastasis in both intratumoral and peritumoral regions (Fig. 1a). Considering that CAFs are the major stromal population widely implicated in the formation of the premetastatic microenvironment in $\mathrm{BCa}^{18}$, we explored the regulatory effect of CAFs in mediating lymphangiogenesis and LN metastasis. As shown in Fig. 1b, alpha-smooth muscle actin (a-SMA)indicated CAFs enrichment in TME was significantly increased in LN metastatic BCa tissues compared with those without LN metastasis. Strikingly, double immunostaining followed by correlation analysis revealed that higher CAF infiltration was related positively to more lymphatic vessel endothelial 
hyaluronan receptor 1 (LYVE1)-indicated MLD in LN metastatic BCa tissues (Fig. 1c-e), suggesting that substantial CAF infiltration in TME is involved in LN metastasis of BCa.

Previous work by our lab uncovered the critical role of IncRNAs in mediating the TME for promoting BCa $L N$ metastasis ${ }^{12}$. Given that tumor-induced $E V s$ are intensively involved in regulating the activation of fibroblasts ${ }^{24}$, we utilized RNA sequencing of urinary EVs from five patients with BCa and five participants without cancer (Gene Expression Omnibus ID: GSE156308) to identify the essential EV-associated IncRNAs contributing to BCa LN metastasis. As shown in Fig. 1f, 255 IncRNAs were upregulated by >2fold in EVs from the patients compared with those from the controls. We then integrated these data with the previous sequencing analysis results of BCa tissues and paired normal adjacent tissues (NATs) as well as LN-positive and LN-negative BCa (GEO: GSE106534). The results showed that the expression of 12 IncRNAs were consistently higher in EVs from the patients than in the controls and in LN-positive than in LN-negative BCa tissues (Supplementary Table 1). Further evaluation of our 228-case BCa cohort demonstrated that BCa tissues markedly overexpressed LINC00665 compared with NATs (Fig. 1g). Consistently, analysis of The Cancer Genome Atlas (TCGA) database demonstrated that LINC00665 was overexpressed in various human cancers and was related to poor prognosis of patients (Fig. 1h, Extended Data Fig. 1a-n). Therefore, LINC00665 was chosen for further analysis.

Next, we assessed the clinical relevance of LINC00665 in BCa. Supplementary Table 2 shows the detailed clinicopathological information of the patients with BCa in our clinical cohort. Quantitative real-time PCR (qRT-PCR) analysis revealed that LINC00665 was overexpressed in LN-positive ( $n=53)$ compared with LN-negative BCa tissues ( $n=175$ ) (Fig. 1i). Metastatic LNs had higher LINC00665 expression levels than primary tumor (Fig. 1j). Importantly, in situ hybridization (ISH) analysis further demonstrated that LINC00665 was greatly overexpressed in BCa tissues with LN metastasis and mildly upregulated in tissues without LN metastasis, whereas it was hardly detected in NATs, suggesting that LINC00665 is closely involved in BCa LN metastasis (Fig. 1k). Moreover, LINC00665 overexpression was associated with shorter overall survival (OS) and disease-free survival (DFS) of the patients with BCa in our clinical cohort (Fig. 1I, 1m). Univariate and multivariate Cox analysis demonstrated that LINC00665 expression was an independent prognostic factor for the OS and DFS of patients with BCa (Supplementary Table 3, 4).

To determine the relationship of LINC00665 with the TME in LN metastatic BCa, we conducted ISH and double immunostaining analysis in BCa patients with LN metastasis. As shown in Fig. $1 \mathrm{n}$ and 10, LINC00665 overexpression related positively to CAF infiltration and around MLD in both the intratumoral and peritumoral regions of LN metastatic BCa tissues, indicating that LINC00665 is associated with CAFinduced $\mathrm{BCa} \mathrm{LN}$ metastasis.

\section{LINC00665 promotes EV production to endow fibroblasts with the CAF phenotype.}

Strikingly, ISH analysis revealed that LN metastatic BCa tissues had a higher extracellular expression level of LINC00665 than those without LN metastasis (Fig. 1k), indicating that extracellular LINC00665 
might play an essential role in BCa LN metastasis. It has been proposed that, as natural nanoscale vesicles in cell-to-cell communication, EVs can carry abnormally expressed IncRNAs through the stromal matrix entering the lymphatic circulation ${ }^{25}$. Therefore, we determined whether LINC00665 exerted its function in BCa LN metastasis via EVs. The culture medium (CM) was collected to isolate and purify the BCa cell-secreted EVs. Transmission electron microscopy (TEM) and nanoparticle tracking analysis (NTA) indicated that LINC00665 overexpression markedly increased the EV concentrations in BCa cells compared with the control (Fig. 2a-c, Extended Data Fig. 2a-e), suggesting that LINC00665 may mediate EV production of BCa cells. Considering that EVs in the BCa TME were prominently internalized by stromal cells and affected their biological activity, we investigated the recipient cells of LINC00665induced EVs. The BCa cell-secreted EVs were labelled with PKH67 and incubated with stromal cells in the BCa TME. Subsequent confocal analysis showed that the LINC00665-induced EVs were mainly internalized by fibroblasts (Fig. 2d). As tumor cell-produced molecular signals, including large

extracellular vesicles, EVs, and cytokines, have been reported to induce stromal cell phenotypic features ${ }^{26}$, we explored the effect of the LINC00665-induced EVs on the fibroblast phenotype by detecting the expression of CAF phenotypic proteins, namely a-SMA and fibroblast activation protein (FAP). As shown in Fig. 2e-h, overexpressing LINC00665 dramatically upregulated a-SMA and FAP expression in the fibroblasts compared with the control, indicating that LINC00665-induced EVs trigger fibroblast transition to the CAF phenotype. Together, these results demonstrate that LINC00665 overexpression promotes EV secretion to endow fibroblasts with the CAF phenotype.

\section{LINC00665 promotes CAF infiltration to mediate BCa lymphangiogenesis and LN metastasis in vitro and} in vivo.

To evaluate the biological function of fibroblasts pretreated with LINC00665-induced EVs, the tube formation and Transwell assays in human lymphatic endothelial cells (HLECs) were performed. As shown in Fig. 2i and 2j, the CM from fibroblasts treated with LINC00665-induced EVs significantly promoted tube formation and migration of HLECs compared with the control, indicating that LINC00665mediated fibroblast transition to CAFs stimulated BCa cell lymphangiogenesis in vitro. Next, we established a xenograft popliteal LN metastasis model in nude mice, as described previously 27,28 , to investigate the effect of LINC00665-induced CAF infiltration on BCa LN metastasis. The EVs were isolated from equal volumes of $\mathrm{CM}$ of BCa cells and verified by NTA assays for intratumoral injection into the primary footpad tumor (Fig. 3a, 3b). Prominently, the LINC00665-induced EVs markedly enhanced the metastasis of luciferase-labeled T24 cells to the popliteal LNs as compared with the control group, and the luminescence of the popliteal LNs gradually increased during the 6-week experiment, as indicated by the In Vivo Imaging System (IVIS) (Fig. 3c, 3d, Extended Data Fig. 2f, 2g). A higher metastatic rate of popliteal LNs in the LINC00665-induced EV group than the control group was observed (Fig. $3 \mathrm{e}-\mathrm{g}$ ), indicating that the LINC00665-induced EV promoted BCa LN metastasis. Moreover, the popliteal LNs of nude mice were enucleated for further IHC staining analysis. As shown in Fig. 3h and $3 \mathrm{i}$, increased CAFs infiltration was observed around the interstitial tissues of popliteal LNs in the LINC00665-induced EV group compared with the control group. Interestingly, double immunofluorescent staining revealed that 
the LINC00665-induced EVs group had consistently increased a-SMA-indicated CAF infiltration and LYVE1-indicated MLD (Fig. 3j-I), confirming that the LINC00665-induced EVs promoted BCa lymphangiogenesis by stimulating CAF infiltration in vivo. Together, these results demonstrate that LINC00665-induced EV secretion promotes CAF infiltration to stimulate BCa lymphangiogenesis and LN metastasis.

\section{LINC00665 directly interacts with heterogeneous nuclear ribonucleoprotein L (hnRNPL).}

To explore the underlying mechanism of LINC00665 in inducing CAF infiltration in $\mathrm{BCa}$, the $5^{\prime}$ and $3^{\prime}$ rapid amplification of CDNA ends (RACE) assays were performed. The results showed that the full-length of LINC00665 was 2856 nucleotides (nt) in BCa cells (Extended Data Fig. 2h-k). Subsequently, fluorescence in situ hybridization (FISH) and subcellular fractionation assays indicated that LINC00665 was located in both the BCa cell cytoplasm and nucleus, but was mainly in the cytoplasm (Extended Data Fig. 2l, 2m). RNA pull-down assay revealed that the biotinylated LINC00665 group had an obviously different band with a molecular weight of 55-70 kDa compared with the control, which was further identified as hnRNPL through Mass spectrometry (MS) analysis (Fig. 4a, 4b). Consistently, western blotting analysis after the RNA pull-down assays demonstrated that LINC00665 specifically enriched hnRNPL (Fig. 4c, 4d). Confocal microscopy analysis validated the colocalization of LINC00665 and hnRNPL in the T24 and 5637 cells (Fig. 4E). RNA immunoprecipitation (RIP) assays verified the significant enrichment of LINC00665 by hnRNPL (Fig. 4f), confirming the interaction between LINC00665 and hnRNPL.

Next, we performed sequential deletion experiments to demonstrate that the 2250-2400 nt region of LINC00665 was essential for its interaction with hnRNPL (Fig. 4g-i). We used POSTAR2, a comprehensive database for exploring the RNA motif for the interaction with RNA-binding proteins ${ }^{29}$, to predict the preferred sequence motif of the hnRNPL binding site that formed a stem-loop structure in the 2285-2360 nt region of LINC00665 (Fig. 4j). Mutation of the 2285-2360 nt region in LINC00665 markedly attenuated the enrichment of LINC00665 by hnRNPL (Fig. 4k), indicating that the region is indispensable for the interaction between LINC00665 and hnRNPL. Taken together, these findings demonstrate the direct interaction between hnRNPL and the 2285-2360 nt region in LINC00665.

\section{LINC00665 promotes RAB27B transcription by forming a DNA-RNA triplex structure with the RAB27B promoter.}

Given that IncRNAs frequently act as molecular drivers of gene transcriptional regulation, leading to tumor initiation and progression ${ }^{30}$, we performed RNA sequencing to profile the target genes of LINC00665. As shown in Fig. 5a, 542 genes were markedly upregulated by $>2$-fold in the LINC00665overexpressing BCa cells compared with control. As LINC00665 widely participates in the release of EVs, the gene expression of the Ras-related Rab protein family associated with EV secretion was analyzed (Fig. 5b, Extended Data Fig. 3a, Supplementary Table 5). The results showed that RAB27B was the most significantly upregulated gene associated positively with LINC00665 overexpression in BCa cells by qRTPCR and western blotting analyses (Fig. 5c, 5d, Extended Data Fig. 3b-e). To explore the potential 
mechanism of LINC00665 in regulating the transcriptional activation of RAB27B, the truncated RAB27B promoter sequences $(-2000$ to $+200 \mathrm{bp}$ ) were cloning into the pGL3 luciferase plasmids and subjected into the luciferase assays. Interestingly, LINC00665 increased the transcriptional activity of the constructs containing the -250 to -500 bp sequence in the RAB27B promoter (Fig. 5e, Extended Data Fig. 3f). Subsequently, chromatin isolation by RNA purification (ChIRP) assays verified that LINC00665 physiologically interacted with the P2 region ( -315 to -327 bp) in the RAB27B promoter in T24 and 5637 cells (Fig. 5f, 5g, Extended Data Fig. 3g). Moreover, a IncRNA-DNA binding motif prediction tool, LongTarget ${ }^{31}$, was used to identify the potential triplex-forming oligonucleotides (TFOs) and corresponding triplex target sites (TTS) in LINC00665 and the RAB27B promoter, among which TFOs were further labeled with 5-carboxy tetramethyl-rhodamine (TAMRA) and TTS with fluorescein amidite (FAM) for the subjection into circular dichroism (CD) spectroscopy and fluorescence resonance energy transfer (FRET) analysis. As shown in Fig. 5h and Extended Data Fig. 3h, the results of CD spectroscopy revealed an obvious positive peak at 270-280 nm and a negative peak at $210 \mathrm{~nm}$ in the LINC00665 TFO2/RAB27B TTS2 group, which was similar to that of the FENDRR/PITX2 positive control ${ }^{32}$. In addition, FRET analysis showed a significant increase in fluorescence intensity at $570-580 \mathrm{~nm}$ and decrease at $520 \mathrm{~nm}$ in the LINC00665 TF02/RAB27B TTS2 group compared with the control group (Fig. 5i, Extended Data Fig. 3i), suggesting the formation of a triplex structure between LINC00665 and the RAB27B promoter.

HnRNPL has been demonstrated as a key regulator in catalyzing H3K4 trimethylation (H3K4me3), the common manner of regulating target gene transcriptional activation ${ }^{33}$. Therefore, we explored whether hnRNPL participates in LINC00665-activated RAB27B transcription by mediating H3K4me3 at the RAB27B promoter in BCa cells. LINC00665 overexpression markedly increased the enrichment of hnRNPL and $\mathrm{H} 3 \mathrm{~K} 4 \mathrm{me} 3$ at the RAB27B promoter, while muting the hnRNPL-binding region on LINC00665 reduced it (Fig. 5j, 5k, Extended Data Fig. 3j, 3k). Conversely, downregulating LINC00665 significantly decreased hnRNPL and H3K4me3 enrichment at the RAB27B promoter (Extended Data Fig. 3I-o). Moreover, silencing hnRNPL greatly impaired the ability of LINC00665 to upregulate RAB27B expression (Fig. 5I, $5 \mathrm{~m})$. Together, these results demonstrate that LINC00665 directly binds to the RAB27B promoter to form a DNA-RNA triplex and activate RAB27B transcription.

\section{LINC00665-induced EVs endow fibroblasts with the CAF phenotype by activating the TGF- $\beta$ pathway.}

Considering that RAB27B is an essential mediator in EV secretion by enhancing the fusion of late endocytic compartments with the plasma membrane ${ }^{34}$, we detected whether RAB27B is crucial for LINC00665-induced EV secretion. The results showed that LINC00665 increased the amount of EVs secreted by T24 and 5637 cells while downregulating RAB27B-impaired LINC00665-induced EV secretion (Fig. $5 n, 5 q$ ). Since RABs were reported to participate in vesicle docking and fusion with recipient cells ${ }^{35}$, we evaluated the essential role of RAB27B underlying fibroblast internalization of LINC00665-induced $\mathrm{EVs}$. As shown in Fig. $5 \mathrm{r}$ and $5 \mathrm{~s}$, an increased green fluorescence signal was observed in the cytoplasm of fibroblasts incubated with PKH67-labeled LINC00665-induced EVs compared with the control, which was 
attenuated by downregulating RAB27B, suggesting that LINC00665 remarkedly enhances fibroblast internalization of BCa cell-secreted EVs via RAB27B.

It was previously reported that TGF- $\beta$ signaling pathway plays a crucial role in regulating the fibroblast phenotype in cancers ${ }^{36}$, we explored the effect of TGF- $\beta$ signaling pathway in fibroblast transition to CAFs as mediated by LINC00665-induced EVs. Analysis of the alterations of crucial proteins in TGF- $\beta$ signaling pathway showed that the phosphorylation of SMAD2 and SMAD3 was markedly increased in fibroblasts treated with LINC00665-induced EVs compared with the control, while fewer change in the expression levels of TGF- $\beta R 1$ and TGF- $\beta$ R2 was observed (Fig. 6a), indicating that LINC00665-induced EVs specifically promoted SMAD2 and SMAD3 phosphorylation to activate the TGF- $\beta$ signaling pathway in fibroblasts. Subsequently, the treatment with SIS3, which specifically inhibits SMAD2 and SMAD3 phosphorylation, was conducted to validate whether TGF- $\beta$ signaling pathway activation is indispensable for the LINC00665-induced EV-mediated phenotype transition of fibroblasts. Immunofluorescence analysis and flow cytometry showed a significant increase in a-SMA and FAP expression in fibroblasts treated with EVs secreted by LINC00665-overexpressing BCa cells, which was markedly attenuated by treating with SIS3 (Fig. 6b-d). Collectively, our findings suggest that LINC00665-induced EVs trigger fibroblast transition to the CAF phenotype by regulating SMAD2 and SMAD3 phosphorylation in the fibroblasts.

\section{LINC00665-induced EVs enhance lymphangiogenesis by stimulating HGF secretion in CAFs.}

To analyze the mechanisms of CAFs activated by LINC00665-induced EV (CAFs $s^{\text {LINC00665-EVs) in }}$ enhancing lymphangiogenesis, we compared the cytokine profiles of CAFs ${ }^{\mathrm{LINC} C 00665-E V s}$ with the control. The results showed that five cytokines were dramatically increased, while one was decreased, in the CM from CAFs ${ }^{\text {LINC00665-EVs }}$ compared with the control (Fig. 6e, 6f). Interestingly, we found that VEGF, the common regulator of lymphangiogenesis, was not significantly secreted by the CAFs $s^{\mathrm{LINC} C 0665-\mathrm{EVS}}$ as compared with the control, indicating that CAFs ${ }^{\mathrm{LINC} C 0665-\mathrm{EVs}}$ might stimulate BCa LN metastasis in a VEGF-independent manner (Fig. 6e, 6f). Moreover, the enzyme-linked immunosorbent assay (ELISA) yielded results consistent with that of the cytokine profiling, in which EVs from LINC00665-silenced BCa cells markedly reduced CAF secretion of cytokines, including IL-6, IL-8, Dkk-1, HGF, and CXCL10, while overexpressing LINC00665 had the opposite effect (Fig. 6G, 6h). Next, in vitro assays were performed to evaluate the role of the above changed cytokines in CAF-induced lymphangiogenesis. The results showed that the treatment with neutralizing antibody against HGF (aHGF) dramatically impaired the tube

formation and migration of HLECs promoted by CAFs ${ }^{\mathrm{LINC} C 0665-\mathrm{EV} s}$, while inhibiting other cytokines had no significant effects (Fig. 6i), suggesting that LINC00665-induced EVs facilitate BCa lymphangiogenesis by stimulating HGF secretion by CAFs.

\section{LINC00665 EV-mediated RAB27B-HGF-c-Myc positive loop is indispensable to BCa LN metastasis.}

Accumulating evidence has shown that tumor cell-stimulated CAFs reciprocally regulate the aggressive biological behavior of tumor cells to support tumor metastasis ${ }^{14}$. Therefore, the biological effect of 
CAFs ${ }^{\text {LINC00665-EVs }}$ in BCa cells was examined. An in vitro coculture model revealed that coculturing with $\mathrm{CAFs}^{\text {LINC00665-EVs }}$ significantly upregulated LINC00665 expression in BCa cells (Fig. 7a, 7b), suggesting the potential formation of a positive feedback loop between BCa cells and CAFs.

It was recently shown that CAFs induce the activation of massive transcription factors in tumor cells to regulate IncRNA transcription ${ }^{13}$. Therefore, we hypothesized that CAFs may induce the transcriptional activation of the LINC00665 promoter to upregulate its expression. To address the hypothesis, we assessed the transcriptional factors that potentially interact with the LINC00665 promoter. The results showed that C-Myc was the most possible transcriptional factor to interact with the LINC00665 promoter, and downregulating c-Myc dramatically reduced the transcriptional activity of LINC00665 promoted by HGF in BCa cells (Fig. 7d, 7e, Extended Data Fig. 4a), confirming that c-Myc widely participates in

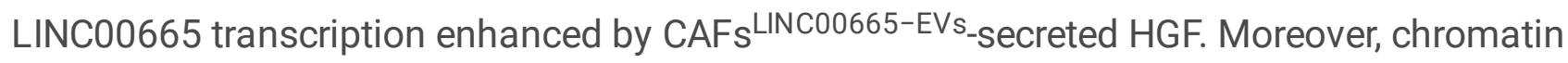
immunoprecipitation (ChIP) analysis demonstrated that CAFs ${ }^{\mathrm{LINC} C 0665-\mathrm{EVS}}$ increased the enrichment of CMyc in the -351 to -358 bp region (referred as P2) of the LINC00665 promoter rather than another predicted binding site located in -106 to -113 bp region (referred as P1) (Fig. 7f-h). Mutating the LINC00665 promoter $\mathrm{P} 2$ region markedly impaired the transcriptional activity increased by the

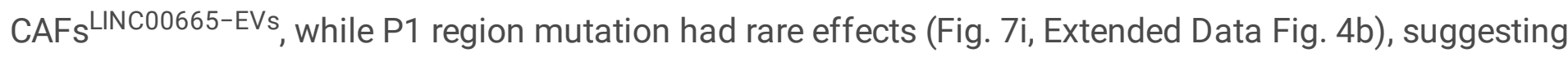

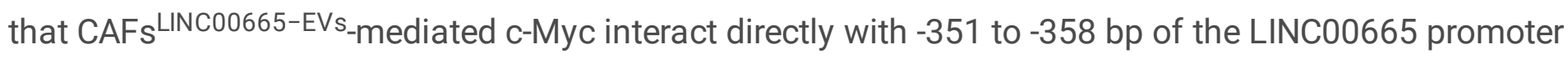
to activate its transcription.

Since CAFs conversely induced LINC00665 transcriptional activation, in vitro assays were performed to detect the role of the LINC00665-mediated positive feedback loop in maintaining CAF-induced lymphangiogenesis (Fig. 7j). The results showed that downregulating LINC00665 expression impaired the fibroblast transition to CAFs induced by coculturing with BCa cells (Fig. 7k-m). Moreover, aHGF treatment significantly blocked CAF-induced LINC00665 overexpression in BCa cells and inhibited the coculturing-induced activation of CAFs (Fig. 7k-m). Strikingly, either LINC00665 silencing or aHGF treatment suppressed the fibroblast-induced tube formation and migration of HLECs in the BCa cell and fibroblast coculture model (Fig. 7n), suggesting that the LINC00665-induced RAB27B-HGF-c-Myc positive feedback loop between $\mathrm{BCa}$ cells and fibroblasts promotes $\mathrm{BCa}$ lymphangiogenesis and $\mathrm{LN}$ metastasis.

\section{Clinical relevance of the LINC00665-induced RAB27B-HGF-c-Myc positive feedback loop in LN metastatic BCa.}

In light of the crucial regulatory role of HGF in LINC00665-induced fibroblast transition to CAFs and BCa lymphangiogenesis, double immunostaining and ISH analysis were conducted to determine the clinical relevance of HGF in BCa LN metastasis. The results showed that higher HGF expression levels were accompanied by more CAF infiltration and MLD in LINC00665-overexpressed regions of LN metastatic BCa tissues (Fig. 8a, 8b). Correlation analysis revealed that HGF overexpression in BCa tissues was associated positively with higher LINC00665 expression (Fig. 8c). Furthermore, qRT-PCR analysis demonstrated that HGF was significantly overexpressed in BCa tissues compared with the paired NATs (n 
= 228) (Fig. 8d). Moreover, LN-positive BCa tissues possessed higher HGF expression levels than the LNnegative BCa tissues (Fig. 8e). Furthermore, HGF overexpression was associated positively with shorter OS and DFS of patients (Fig. 8f, 8g).

To assess the effect of blocking HGF in suppressing lymphangiogenesis and LN metastasis mediated by the LINC00665-induced RAB27B-HGF-c-Myc positive feedback loop, in vitro coculture model and in vivo popliteal LN metastasis model was conducted. In vitro assays showed that both fibroblasts treated with LINC00665-induced EVs and primary CAFs dramatically promoted tube formation and migration of HLECs, while aHGF treatment dramatically abrogated these effects (Fig. 8h, Extended Data Fig. 4d-e). Moreover, in vivo popliteal LN metastasis assays demonstrated that LINC00665-induced EVs significantly promoted BCa cells to metastasize to the LNs compared with the control groups, whereas HGF inhibition dramatically reversed this effect (Fig. 6i, 6j). Statistical analysis showed that aHGF significantly attenuated the LN metastatic rate increased by the LINC00665-induced EVs (Extended Data Fig. 4f). Furthermore, blockage of HGF reduced the CAF infiltration and MLD mediated by the LINC0065-induced EVs in the tumor tissues (Fig. 8k).

The previous study has demonstrated that Cabozantinib, a small-molecule inhibitor specifically targeting the HGF-MET signaling pathway, exhibits a noticeable clinical effect in suppressing tumor angiogenesis ${ }^{7}$. As the essential role of HGF in BCa lymphangiogenesis and LN metastasis has been wellelucidated in a large clinical cohort and in vitro and in vivo experiments, we further constructed an orthotopic xenograft model to evaluate the clinical application potential of cabozantinib in LINC00665mediated lymphangiogenesis and LN metastatic BCa (Fig. 8I). The results showed that the utilization of Cabozantinib decreased the orthotopic tumor volume in nude mice educated by LINC00665-induced EVs as compared with the control group (Fig. $8 \mathrm{~m}$ ). Importantly, cabozantinib combined with cisplatin, the main chemotherapeutic drug for advanced $\mathrm{BCa}^{5}$, showed a significant effect in reducing tumor volume and prolonging the tumor-bearing survival time of LINC00665-induced EV-educated mice (Fig. 8m, 8n), revealing the encouraging clinical potential of cabozantinib in treating LINC00665-induced LN metastatic $\mathrm{BCa}$. Taken together, these results demonstrate that the LINC00665-induced RAB27B-HGF-c-Myc positive feedback loop between BCa cells and fibroblasts plays a significant role in BCa LN metastasis (Figure 80).

\section{Discussion}

CAF infiltration marks an aggressive signature in the TME, which orchestrates the metastasis of malignancies through complex interactions with cancer cells ${ }^{37}$. It has been reported that the activation of $\mathrm{CAF}$ in $\mathrm{BCa}$ is accompanied by the invasive behavior of endothelial cells that influence tumor angiogenesis ${ }^{38}$. However, the role and mechanism of CAFs in regulating lymphangiogenesis to trigger BCa LN metastasis remain largely unknown. Here, we demonstrate that EV-associated LINC00665 mediated the EV secretion by $\mathrm{BCa}$ cells to foster $\mathrm{CAF}$ infiltration and to facilitate lymphangiogenesis in BCa. LINC00665 recruited hnRNPL to mediate the RAB27B-dependent release of EVs that allowed fibroblasts to acquire the CAF phenotype, thereby fostering lymphangiogenesis and LN metastasis in 
BCa. Our findings highlight for the first time that an EV-associated IncRNA acts as a key mediator for communication between cancer cells and fibroblasts during BCa LN metastasis, suggesting that targeting LINC00665 is a potential approach to selectively suppress CAF infiltration and inhibiting BCa LN metastasis.

Here, we determined that the production of HGF sustained by a LINC00665-induced RAB27B-HGF-c-Myc positive feedback loop between cancer cells and fibroblasts contributed to BCa lymphangiogenesis and LN metastasis. The current therapy targeting HGF signaling in the clinic is mainly based on the application of a widely used HGF-Met pathway inhibitor, cabozantinib, which exhibits potent inhibitory activity against angiogenesis for the treatment of non-small-cell lung cancer, liver cancer, and renal cancer $^{39-41}$. Nevertheless, evidence for the effect of cabozantinib on lymphangiogenesis is still lacking. In the present study, using an orthotopic xenograft model, we first report that cabozantinib significantly enhanced the efficacy in reducing the tumor burden and prolonging the survival time in combination with cisplatin, a widely used chemotherapy for metastatic $\mathrm{BCa}^{5}$. These findings provide preliminary evidence for HGF-targeting therapy in LN metastatic BCa. Further clinical studies are required for the application of cabozantinib in $\mathrm{BCa}$ with $\mathrm{LN}$ metastasis, presenting a promising prospect for broadening the indications for cabozantinib.

The release of EVs is believed to be controlled by RAB proteins ${ }^{42}$. RABs predominantly participate in endomembrane trafficking and modulate the budding efficiency of membrane-wrapped vesicles as $\mathrm{EVs}^{35}$. Recent studies have indicated that the type of functional RAB may influence the role of EVs during a variety of biological events ${ }^{43}$. Nevertheless, understanding of the core regulator triggered by EV signaling in BCa LN metastasis is incomplete. Herein, through RNA-sequencing followed by analysis of RAB expression patterns, we determined that RAB27B correlates specifically with LINC00665-induced lymphangiogenesis-driven EV secretion. Further, we found that the overexpression of RAB27B during the LN metastasis of BCa was caused by LINC00665-mediated transcription activation, in which LINC00665 induced H3K4me3 modification on the promoter of RAB27B through the recruitment of hnRNPL. Moreover, RAB27B-induced EV secretion promoted BCa lymphangiogenesis and LN metastasis. These findings reveal the pivotal role of RAB27B underlying LN metastasis, expanding our knowledge of RABmediated $\mathrm{EV}$ regulation in $\mathrm{LN}$ metastatic $\mathrm{BCa}$.

Although a variety of functional molecules have been identified as potential therapeutic targets in $\mathrm{BCa}$, in vivo treatment faces great challenges due to their instability and the existence of biological barriers ${ }^{5}$. Recent evidence shows that EVs are promising for molecular therapy in cancers due to their high histocompatibility and capacity for crossing biological barriers ${ }^{44,45}$. Therefore, controlling EV biogenesis and delivery to enable molecule transmission represents the predominant process for achieving therapeutic goals. In the present study, we determined that the EV-associated IncRNA LINC00665 was involved in the production of EVs that targeting fibroblasts. Moreover, inhibiting LINC00665 significantly blocked the delivery of fibroblast-targeted EVs, thus effectively suppressing BCa lymphangiogenesis and LN metastasis in animal experiments. Further exploration of the application of LINC00665 in the 
emerging technologies that modify EVs to improve the yield, targeting efficiency, and content of beneficial cargos may develop a feasible therapeutic intervention strategy against BCa LN metastasis.

In summary, we elucidate a novel mechanism underlying EV-associated IncRNA-mediated CAF infiltration to facilitate the LN metastasis of BCa, in which the LINC00665-induced RAB27B-HGF-c-Myc positive feedback loop between cancer cells and fibroblasts sustains lymphangiogenesis. Our study highlights LINC00665-driven HGF signaling as a promising therapeutic target and demonstrates that utilization of the HGF-MET inhibitor cabozantinib is an encouraging treatment for LN metastatic BCa.

\section{Methods}

\section{Patients and clinical samples.}

All BCa tissues and NATs in this study were obtained with consent from patients who had undergone surgery at the Sun Yat-Sen Memorial Hospital of Sun Yat-Sen University and with the approval of the Sun Yat-Sen University Committees for Ethical Review of Research involving Human Subjects. The tissues were pathologically diagnosed with $\mathrm{BCa}$ independently by two pathologists and underwent total RNA extraction, or formalin fixation and paraffin embedding for immunostaining.

\section{Cell lines and cell culture.}

The T24 and 5637 human BCa cell lines were purchased from American Type Culture Collection (Manassas, VA, USA) and were cultured in Roswell Park Memorial Institute (RPMI) 1640 medium (Invitrogen, Waltham, MA, USA). Human fibroblasts were acquired from the National Collection of Authenticated Cell Cultures (Shanghai, China) and maintained in minimal essential medium (MEM; Invitrogen). HLECs were obtained from ScienCell Research Laboratories (Carlsbad, CA, USA) and cultured in endothelial cell medium (ECM; ScienCell Research Laboratories). The ECM was supplemented with $5 \%$ fetal bovine serum (FBS); the other culture media were supplemented with $10 \%$ FBS. All cells were cultured in a humidified incubator with $5 \% \mathrm{CO}_{2}$ at $37^{\circ} \mathrm{C}$.

\section{Cell transfection and lentivirus-mediated transduction.}

For cell transfection, the small interfering RNA (siRNA) oligonucleotides for the target gene and the pcDNA3.1 expression vector with the target gene were purchased from GenePharma (Shanghai, China) and were used to knock down or overexpress the target gene in $\mathrm{BCa}$ cells, respectively. All transfections were conducted with assistance from Lipofectamine 3000 (Invitrogen) under the manufacturer's protocols. Transfection efficiency was evaluated using qRT-PCR.

For lentivirus infection, we first packaged the LINC00665 into lentivirus vector (GenePharma, Shanghai, China), which was transfected into HEK-293T cells to amplify the target virus. Then, the lentiviruses were harvested and purified for infecting the BCa cells to construct the LINC00665 stable overexpression cell 
lines. Successfully infected cells were selected with puromycin (Sigma-Aldrich, St. Louis, MO, USA) over 2 weeks.

\section{Isolation and purification of EVs.}

The EVs were isolated from the cell culture medium using differential centrifugation, where the cell culture medium after 48-h incubation with EV-depleted FBS was collected and centrifuged at 2,000 $\times g$ for $10 \mathrm{~min}, 10,000 \times g$ for $30 \mathrm{~min}$, and $120,000 \times g$ for $70 \mathrm{~min}$. Then, the pellet was resuspended in PBS, followed by centrifugation at $120,000 \times g$ for $70 \mathrm{~min}$, after which the isolated pellet was resuspended in proper PBS. The purified EVs were stored at $-80^{\circ} \mathrm{C}$ until further use.

\section{Flow cytometry analysis of fibroblast phenotype.}

The cells were harvested and washed with PBS three times, followed by 30-min incubation with primary antibodies at $4^{\circ} \mathrm{C}$ and with the corresponding secondary fluorescent antibody at $4^{\circ} \mathrm{C}$ for another $30 \mathrm{~min}$. Subsequently, the treated cells were analyzed using a FACScan flow cytometer (Becton Dickinson, Brea, CA, USA).

\section{Measurement of LINC00665-induced EVs on LN metastasis of BCa cells.}

All experimental procedures were performed with the approval of the Sun Yat-Sen University Institutional Animal Care and Use Committee. Here, we used nude mice (4-6 weeks old, 18-20 g) purchased from the Sun Yat-Sen University Experimental Animal Center (Guangzhou, China). As for mice popliteal lymphatic metastasis model, luciferase-labeled T24 cells were implanted into the footpads of the mice, which were then randomly divided into different groups $(n=12)$, followed by intratumoral co-injection with EVs secreted by the T24 cells. LN metastasis was detected using a PerkinElmer IVIS Spectrum In Vivo Imaging System. CAF infiltration in the primary tumors was analyzed with IHC as described in Supplementary Method.

For the orthotopic xenograft model, the mice were anesthetized with $4 \%$ chloral hydrate. Then, an angiocatheter (24-gauge; Terumo Medical Products) lubricated with paraffin oil was gently inserted into the urethra to remove the urine and inoculate $5 \times 10^{5} \mathrm{~T} 24$ cells suspended in $50 \mu \mathrm{L}$ medium into the bladder. The catheter was left in place for $60 \mathrm{~min}$. After the orthotopic xenograft model had been constructed, the mice were randomly divided into three groups, followed by treatment with LINC00665induced EVs with or without cabozantinib and cisplatin.

\section{Verification of the binding between LINC00665 and hnRNPL.}

The proteins interacting with LINC00665 were detected using RNA pull-down assays. Briefly, biotinlabelled LINC00665 and antisense were synthesized according to the instructions of the transcription kit (Thermo Fisher Scientific, Waltham, MA, USA). Then, the extracted nuclear proteins or recombinant hnRNPL underwent biotin-labelled LINC00665 pull-down assays using a Pierce Magnetic RNA-Protein 
Pull-Down Kit (Thermo Fisher Scientific) to determine the direct interaction between LINC00665 and hnRNPL. Subsequently, the eluted proteins were evaluated via silver staining or western blotting analysis.

For detecting the hnRNPL-binding region on LINC00665, a series of vectors containing different lengths of the LINC00665 sequence were constructed and subjected to RNA pull-down assays as described above.

\section{Detection of physiological interaction between LINC00665 and the RAB27B promoter.}

The interaction between LINC00665 and the RAB27B promoter was evaluated by ChIRP assays conducted using the Magna ChIRP RNA Interactome Kit (Millipore, Billerica, MA, USA). Briefly, $2 \times 10^{7}$ indicated $\mathrm{BCa}$ cells were fixed in $1 \%$ formaldehyde and lysed to obtain the nuclear extracts. Then, the chromatin was sheared with ultrasonication, and the sonicated lysate was incubated with biotin-labelled LINC00665 probes for $4 \mathrm{~h}$ at $37^{\circ} \mathrm{C}$. The targeted chromatin was pulled down with streptavidin magnetic beads and subjected to qRT-PCR analysis to examine the enrichment of RAB27B promoter.

\section{FRET and CD spectroscopy.}

To determine the formation of the triplex structure between LINC00665 and the RAB27B promoter, we performed FRET and CD spectroscopy. For the FRET assays, the TFOs and TTS were labeled with TAMRA and FAM, respectively. Then, 1:5 TFOs and TTS were mixed in binding buffer, followed by incubation at $55^{\circ} \mathrm{C}$ for $10 \mathrm{~min}$ and $37^{\circ} \mathrm{C}$ for $10 \mathrm{~h}$. The fluorescence wavelengths between 480 and $690 \mathrm{~nm}$ were measured with a Molecular Device M5 Plate Reader (USA).

For CD spectroscopy, 1:1 TFOs and TTS were mixed in binding buffer and equilibrated at $30^{\circ} \mathrm{C}$ for $1 \mathrm{~h}$. Then, the fluorescence wavelengths were measured with a Chirascan spectrometer. Supplementary Table 5 lists the oligos used in the FRET and CD spectroscopy.

\section{Cytokine profiling of EV-induced fibroblasts.}

The cytokine secretion profile of the EV-induced fibroblasts was detected using a Proteome Profiler Human XL Cytokine Array Kit (R\&D Systems, Minneapolis, MN, USA). The cell culture supernatants from EV-pretreated fibroblasts were collected and incubated overnight at $4^{\circ} \mathrm{C}$ on antibody-containing nitrocellulose membranes. Then, the detection antibody cocktail was added for 1 -h incubation at room temperature, followed with 30-min treatment with streptavidin-horseradish peroxidase. Finally, the cytokine expression profile was visualized with Chemi Reagent Mix (Thermo Fisher Scientific, Waltham, MA, USA), and recorded with X-ray film.

\section{Bioinformatic analysis.}

The clinical role of LINC00665 in human cancers was analyzed through TCGA database at GEPIA (http://gepia.cancer-pku.cn/index.html). The secondary structures of LINC00665 and the hnRNPL binding 
motif were predicted in RNAalifold (http://rna.tbi.univie.ac.at/cgi-bin/RNAWebSuite/RNAalifold.cgi) and POSTAR3 (http://postar.ncrnalab.org/), respectively.

\section{Statistical analysis.}

All statistical analyses were conducted using SPSS 13 (SPSS Inc., Chicago, IL, USA) from at least three independent experiments, in which $P<0.05$ was considered statistically significant. Quantitative data are presented as the means \pm standard deviations (SD). The statistical significance of nonparametric variables was analyzed with the chi-square test $\left(\chi^{2}\right.$ test); that for parametric variables was analyzed with the 2-tailed Student's t test or 1-way analysis of variance (ANOVA). The patients' OS and DFS were analyzed with the Kaplan-Meier method.

\section{Declarations}

\section{Acknowledgements}

We thank Prof. JX Zhang of the Department of Medical Statistics and Epidemiology, Sun Yat-Sen University, for statistical advice and research comments. This study was funded by the National Key Research and Development Program of China (Grant No. 2018YFA0902803); the National Natural Science Foundation of China (Grant No. 82173271, 82173272, 81825016, 81802530, 82103536, 81871945 and 81902589); Guangdong Basic and Applied Basic Research Foundation (Grant No. 2021B1515020091, 2020A1515010815, 2018B010109006, and 2021A1515010355); and the Science and Technology Program of Guangzhou, China (Grant No. 202002030388, 201803010049, and 2017B020227007).

\section{Author contributions}

$\mathrm{CHC}$ and $\mathrm{JH}$ contributed to the design of the study. $\mathrm{YML}, \mathrm{HHZ}$, and $\mathrm{YK}$ conducted the in vitro and in vivo assays. YTL, YZ, and HH carried out the clinical data analyses. YL and ZHL performed the ISH and IHC analysis. YNY, MJA, and HH conducted the qRT-PCR and western blotting analysis. YYY, MJA, and YL conducted the mechanistic experiments. $\mathrm{CHC}, \mathrm{YML}$, and $\mathrm{HHZ}$ wrote the manuscript. All authors have read and approved the final manuscript.

\section{Competing interests statement}

The authors declare no potential conflicts of interest. 


\section{Abbreviations}

CAFs, Cancer-associated fibroblasts; LN, lymph node; BCa, bladder cancer; EVs, extracellular vesicles; NMIBC, non-muscle-invasive bladder cancer; MIBC, muscle-invasive bladder cancer; TME, tumor microenvironment; IncRNAs, long noncoding RNAs; IHC, immunohistochemistry; MLD, microlymphatic vessel density; a-SMA, alpha-Smooth Muscle Actin; LYVE1, lymphatic vessel endothelial hyaluronan receptor 1; NATs, normal adjacent tissues; TCGA, The Cancer Genome Atlas; qRT-PCR, quantitative realtime PCR; ISH, in situ hybridization; OS, overall survival; DFS, disease-free survival; CM, culture media; TEM, transmission electron microscopy; NTA, nanoparticle tracking analysis; FAP, fibroblast activation protein; HLECs, human lymphatic endothelial cells; IVIS, In Vivo Imaging System; hnRNPL, heterogeneous nuclear ribonucleoprotein L; RACE, 5' and 3' rapid amplification of cDNA ends; nt, nucleotides; FISH, fluorescence in situ hybridization; MS, Mass spectrometry; RIP, RNA immunoprecipitation; ChIRP, chromatin isolation by RNA purification; TFOs, triplex-forming oligonucleotides; TTS, triplex target sites; TAMRA, tetramethyl-rhodamine; FAM, fluorescein amidite; CD, circular dichroism; FRET, fluorescence

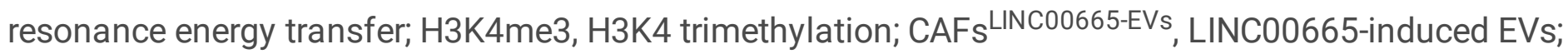
ELISA, enzyme-linked immunosorbent assay; aHGF, neutralizing antibody against HGF; ChIP, chromatin immunoprecipitation; NSCLC, non-small-cell lung cancer; ATCC, American Type Culture Collection; RPMI, Roswell Park Memorial Institute medium; MEM, Minimal Essential Medium; ECM, Endothelial Cell Medium; FBS, fetal bovine serum; SD, standard deviations; $\chi 2$ test, chi-square test;

\section{References}

1. Bray, F. et al. Global cancer statistics 2018: GLOBOCAN estimates of incidence and mortality worldwide for 36 cancers in 185 countries. CA Cancer J Clin 68, 394-424, doi: 10.3322/caac.21492 (2018).

2. Cumberbatch, M. G. K. et al. Epidemiology of Bladder Cancer: A Systematic Review and Contemporary Update of Risk Factors in 2018. Eur Urol 74, 784-795, doi:

10.1016/j.eururo.2018.09.001 (2018).

3. Bruins, H. M. et al. The impact of the extent of lymphadenectomy on oncologic outcomes in patients undergoing radical cystectomy for bladder cancer: a systematic review. Eur Urol 66, 1065-1077, doi: 10.1016/j.eururo.2014.05.031 (2014).

4. Lenis, A. T., Lec, P. M., Chamie, K. \& Mshs, M. D. Bladder Cancer: A Review. JAMA 324, 1980-1991, doi: 10.1001/jama.2020.17598 (2020).

5. Cathomas, R. et al. The 2021 Updated European Association of Urology Guidelines on Metastatic Urothelial Carcinoma. Eur Urol, doi: 10.1016/j.eururo.2021.09.026 (2021).

6. Rosenberg, J. E. et al. Randomized Phase III Trial of Gemcitabine and Cisplatin With Bevacizumab or Placebo in Patients With Advanced Urothelial Carcinoma: Results of CALGB 90601 (Alliance). J Clin Onco/ 39, 2486-2496, doi:10.1200/JC0.21.00286 (2021). 
7. Fallahi, P. et al. Molecular targets of tyrosine kinase inhibitors in thyroid cancer. Semin Cancer Biol, doi:10.1016/j.semcancer.2020.11.013 (2020).

8. Escobedo, N. \& Oliver, G. Lymphangiogenesis: Origin, Specification, and Cell Fate Determination. Annu Rev Cell Dev Bio/ 32, 677-691, doi:10.1146/annurev-cellbio-111315-124944 (2016).

9. Alitalo, K. \& Carmeliet, P. Molecular mechanisms of lymphangiogenesis in health and disease. Cancer Cell 1, 219-227, doi:10.1016/s1535-6108(02)00051-x (2002).

10. Chen, C. et al. Exosomal long noncoding RNA LNMAT2 promotes lymphatic metastasis in bladder cancer. J Clin Invest 130, 404-421, doi:10.1172/JCl130892 (2020).

11. Munir, H., Mazzaglia, C. \& Shields, J. D. Stromal regulation of tumor-associated lymphatics. Adv Drug Deliv Rev 161-162, 75-89, doi:10.1016/j.addr.2020.08.001 (2020).

12. Chen, C. et al. LNMAT1 promotes lymphatic metastasis of bladder cancer via CCL2 dependent macrophage recruitment. Nat Commun 9, 3826, doi:10.1038/s41467-018-06152-x (2018).

13. Biffi, G. \& Tuveson, D. A. Diversity and Biology of Cancer-Associated Fibroblasts. Physiol Rev 101, 147-176, doi:10.1152/physrev.00048.2019 (2021).

14. Chen, Y., McAndrews, K. M. \& Kalluri, R. Clinical and therapeutic relevance of cancer-associated fibroblasts. Nat Rev Clin Oncol, doi:10.1038/s41571-021-00546-5 (2021).

15. Bulle, A. \& Lim, K. H. Beyond just a tight fortress: contribution of stroma to epithelial-mesenchymal transition in pancreatic cancer. Signal Transduct Target Ther 5, 249, doi:10.1038/s41392-020-003411 (2020).

16. Lin, Y. et al. CAFs shape myeloid-derived suppressor cells to promote stemness of intrahepatic cholangiocarcinoma via 5-lipoxygenase. Hepatology, doi:10.1002/hep.32099 (2021).

17. Mao, X. et al. Crosstalk between cancer-associated fibroblasts and immune cells in the tumor microenvironment: new findings and future perspectives. Mol Cancer 20, 131, doi:10.1186/s12943021-01428-1 (2021).

18. Chen, Z. et al. Single-cell RNA sequencing highlights the role of inflammatory cancer-associated fibroblasts in bladder urothelial carcinoma. Nat Commun 11, 5077, doi:10.1038/s41467-020-18916-5 (2020).

19. Morrissey, S. M. et al. Tumor-derived exosomes drive immunosuppressive macrophages in a premetastatic niche through glycolytic dominant metabolic reprogramming. Cell Metab 33, 2040-2058 e2010, doi:10.1016/j.cmet.2021.09.002 (2021).

20. Xi, L. et al. Hypoxia-stimulated ATM activation regulates autophagy-associated exosome release from cancer-associated fibroblasts to promote cancer cell invasion. J Extracell Vesicles 10, e12146, doi:10.1002/jev2.12146 (2021).

21. Gao, Y. et al. CD63(+) Cancer-Associated Fibroblasts Confer Tamoxifen Resistance to Breast Cancer Cells through Exosomal miR-22. Adv Sci (Weinh) 7, 2002518, doi:10.1002/advs.202002518 (2020).

22. Liu, S. J., Dang, H. X., Lim, D. A., Feng, F. Y. \& Maher, C. A. Long noncoding RNAs in cancer metastasis. Nat Rev Cancer 21, 446-460, doi:10.1038/s41568-021-00353-1 (2021). 
23. Karaman, S. \& Detmar, M. Mechanisms of lymphatic metastasis. J Clin Invest 124, 922-928, doi:10.1172/JCI71606 (2014).

24. Paggetti, J. et al. Exosomes released by chronic lymphocytic leukemia cells induce the transition of stromal cells into cancer-associated fibroblasts. Blood 126, 1106-1117, doi:10.1182/blood-2014-12618025 (2015).

25. Mathieu, M., Martin-Jaular, L., Lavieu, G. \& Thery, C. Specificities of secretion and uptake of exosomes and other extracellular vesicles for cell-to-cell communication. Nat Cell Bio/ 21, 9-17, doi:10.1038/s41556-018-0250-9 (2019).

26. Xu, R. et al. Extracellular vesicles in cancer - implications for future improvements in cancer care. Nat Rev Clin Oncol 15, 617-638, doi:10.1038/s41571-018-0036-9 (2018).

27. Zheng, H. et al. Tumor-derived exosomal BCYRN1 activates WNT5A/VEGF-C/VEGFR3 feedforward loop to drive lymphatic metastasis of bladder cancer. Clin Trans/ Med 11, e497, doi:10.1002/ctm2.497 (2021).

28. Zhu, J. et al. circEHBP1 promotes lymphangiogenesis and lymphatic metastasis of bladder cancer via miR-130a-3p/TGFbetaR1/VEGF-D signaling. Mol Ther, doi:10.1016/j.ymthe.2021.01.031 (2021).

29. Zhu, Y. et al. POSTAR2: deciphering the post-transcriptional regulatory logics. Nucleic Acids Res 47, D203-D211, doi:10.1093/nar/gky830 (2019).

30. Schmitt, A. M. \& Chang, H. Y. Long Noncoding RNAs in Cancer Pathways. Cancer Cell 29, 452-463, doi:10.1016/j.ccell.2016.03.010 (2016).

31. He, S., Zhang, H., Liu, H. \& Zhu, H. LongTarget: a tool to predict IncRNA DNA-binding motifs and binding sites via Hoogsteen base-pairing analysis. Bioinformatics 31, 178-186, doi:10.1093/bioinformatics/btu643 (2015).

32. Grote, P. \& Herrmann, B. G. The long non-coding RNA Fendrr links epigenetic control mechanisms to gene regulatory networks in mammalian embryogenesis. RNA Biol 10, 1579-1585, doi:10.4161/rna.26165 (2013).

33. Atianand, M. K. et al. A Long Noncoding RNA lincRNA-EPS Acts as a Transcriptional Brake to Restrain Inflammation. Cell 165, 1672-1685, doi:10.1016/j.cell.2016.05.075 (2016).

34. Ostrowski, M. et al. Rab27a and Rab27b control different steps of the exosome secretion pathway. Nat Cell Biol 12, 19-30; sup pp 11-13, doi:10.1038/ncb2000 (2010).

35. Jin, H. et al. Rab GTPases: Central Coordinators of Membrane Trafficking in Cancer. Front Cell Dev Bio/ 9, 648384, doi:10.3389/fcell.2021.648384 (2021).

36. Calon, A., Tauriello, D. V. \& Batlle, E. TGF-beta in CAF-mediated tumor growth and metastasis. Semin Cancer Biol 25, 15-22, doi:10.1016/j.semcancer.2013.12.008 (2014).

37. Barrett, R. \& Pure, E. Cancer-associated fibroblasts: key determinants of tumor immunity and immunotherapy. Curr Opin Immunol 64, 80-87, doi:10.1016/j.coi.2020.03.004 (2020).

38. Miyake, M. et al. CXCL1-Mediated Interaction of Cancer Cells with Tumor-Associated Macrophages and Cancer-Associated Fibroblasts Promotes Tumor Progression in Human Bladder Cancer. 
Neoplasia 18, 636-646, doi:10.1016/j.neo.2016.08.002 (2016).

39. Gautschi, O. et al. Targeting RET in Patients With RET-Rearranged Lung Cancers: Results From the Global, Multicenter RET Registry. J Clin Onco/35, 1403-1410, doi:10.1200/JC0.2016.70.9352 (2017).

40. Shang, R. et al. Cabozantinib-based combination therapy for the treatment of hepatocellular carcinoma. Gut 70, 1746-1757, doi:10.1136/gutjnl-2020-320716 (2021).

41. Hirsch, L. et al. Clinical Activity and Safety of Cabozantinib for Brain Metastases in Patients With Renal Cell Carcinoma. JAMA Oncol, doi:10.1001/jamaoncol.2021.4544 (2021).

42. Colombo, M., Raposo, G. \& Thery, C. Biogenesis, secretion, and intercellular interactions of exosomes and other extracellular vesicles. Annu Rev Cell Dev Bio/ 30, 255-289, doi:10.1146/annurev-cellbio101512-122326 (2014).

43. Wang, F. W. et al. APC-activated long noncoding RNA inhibits colorectal carcinoma pathogenesis through reduction of exosome production. Journal of Clinical Investigation 129, 727-743, doi:10.1172/Jci122478 (2019).

44. Alvarez-Erviti, L. et al. Delivery of siRNA to the mouse brain by systemic injection of targeted exosomes. Nat Biotechnol 29, 341-345, doi:10.1038/nbt.1807 (2011).

45. Azmi, A. S., Bao, B. \& Sarkar, F. H. Exosomes in cancer development, metastasis, and drug resistance: a comprehensive review. Cancer Metastasis Rev 32, 623-642, doi:10.1007/s10555-013-9441-9 (2013).

\section{Figures}




\section{Figure 1}

LINC00665 overexpression is associated with CAF-induced LN metastasis of BCa. a, Representative IHC images and percentages of LYVE1-indicated lymphatic vessel density in BCa tissues. Scale bar: $50 \mu \mathrm{m}$. Statistical significance was assessed by the $\chi 2$ test. $b$, Representative $\mathrm{IHC}$ images and percentages of aSMA-indicated CAF infiltration in NATs and BCa tissues with or without LN metastasis. Scale bar: $50 \mu \mathrm{m}$. Statistical significance was assessed by the $\chi 2$ test. $\mathrm{C}-\mathrm{e}$, Representative $\mathrm{IHC}$ images and percentages 
revealing a positive correlation between a-SMA-indicated CAF infiltration and LYVE1-indicated lymphatic vessel density in BCa tissues. Scale bar: $50 \mu \mathrm{m}$. Statistical significance was assessed by the $\chi 2$ test. $\mathrm{f}$, Schematic diagram of the screening of IncRNAs upregulated in both urinary EVs and LN-positive tissues from patients with BCa. g, h, Analysis of LINC00665 expression in BCa tissues compared with NATs in a 228-case cohort $(\mathrm{g})$ and TCGA database $(\mathrm{h})$. Statistical significance was assessed by the nonparametric Mann-Whitney $\mathrm{U}$ test. i, j, qRT-PCR analysis of LINC00665 expression between LN-positive BCa tissues and LN-negative BCa tissues (i) and between primary tumors and paired metastatic LNs (j) from patients with BCa. Statistical significance was assessed by the nonparametric Mann-Whitney U test. k, Representative ISH images and percentages of LINC00665 expression (blue) in NATs and BCa with or without LN metastasis. Scale bar: $50 \mu \mathrm{m}$. Statistical significance was assessed by the $\chi 2$ test. I, $\mathrm{m}$, Kaplan-Meier survival analysis of the OS (I) or DFS (m) of patients with BCa according to LINC00665 expression. The cutoff value is the median expression. $n, 0$, Representative images and percentages of $\mathrm{CAF}$ infiltration and lymphatic vessel density in the intratumoral $(\mathrm{n})$ and peritumoral regions (o) of $\mathrm{BCa}$ tissues according to LINC00665 expression. Scale bar: $50 \mu \mathrm{m}$. Statistical significance was assessed by the $\chi 2$ test. $* P<0.05, * * P<0.01$. 


\section{Figure 2}

LINC00665 promotes EV production to endow fibroblasts with the CAF phenotype in vitro. a, Representative TEM images of EVs isolated from BCa cells with or without LINC00665 overexpression. Scale bars: $100 \mathrm{~nm}$. b, c, NTA analysis and quantification of EVs isolated from BCa cells with or without LINC00665 overexpression. d, Representative fluorescence images of fibroblasts, macrophages, $T$ cells, and B cells after incubation with PKH67-labelled (green) in vector-induced EVs or LINC00665-induced EVs from BCa cells. Scale bars: $5 \mu \mathrm{m}$. e, Western blotting analysis of a-SMA and FAP expression in fibroblasts incubated with vector- or LINC00665-induced EVs from BCa cells. f, Representative confocal images of a- 
SMA and FAP expression in fibroblasts incubated with vector- or LINC00665-induced EVs from BCa cells. Scale bars: $5 \mu \mathrm{m} . \mathrm{g}$, h, Flow cytometric analysis and quantification of a-SMA (g) or FAP (h) expression in fibroblasts treated with PBS, vector-induced EVs, or LINC00665-induced EVs form BCa cells. i, j, Representative images and quantification of tube formation (i) and Transwell migration (j) of HLECs incubated with CM from PBS, vector-induced EVs, or LINC00665-induced EV-treated fibroblasts.

Statistical significance was assessed by the two-tailed Student's $t$ test in d or 1-way ANOVA followed by Dunnett's tests in $\mathrm{h}$ and $\mathrm{j}$. Error bars show the SDs from three independent experiments. ${ }^{\star} \mathrm{P}<0.05$; ${ }^{\star *} \mathrm{P}<$ 0.01 . 


\section{Figure 3}

LINC00665 promotes CAF infiltration to mediate LN metastasis of BCa in vivo. a, Schematic illustration of the establishment of the popliteal LN metastasis model. b, NTA analysis of EVs isolated from T24 cells with or without LINC00665 overexpression. c, Representative bioluminescence images of the metastatic popliteal LN of nude mice treated with vector-induced EVs or LINC00665-induced EVs $(n=12)$. The red arrows indicate footpad primary tumor and metastatic popliteal LN. d, Representative images showing 
the popliteal LNs of the mice $(n=12)$. e, Representative images of IHC analysis of anti-luciferase popliteal LNs in indicated group $(n=12)$. Scale bars: $500 \mu \mathrm{m}$ (red) or $50 \mu \mathrm{m}$ (black). $\mathrm{f}$, Assessment of the popliteal $L N$ metastatic rate $(n=12)$. Statistical significance was assessed by the $\chi 2$ test. $g$, Quantification of the popliteal LN volume of the mice in indicated group $(n=12) . h$, i, Representative images and histogram analysis of IHC staining of a-SMA-indicated CAF infiltration in the popliteal LNs in indicated group $(n=12)$. Scale bars: $50 \mu \mathrm{m}$. $j-I$ Representative images and quantification of fluorescence of a-SMA-indicated CAF and LYVE1-indicated lymphatic vessel density in footpad tumor from the mice ( $\mathrm{n}$ $=12$ ). Scale bar: $5 \mu \mathrm{m}$. Statistical significance was assessed by the two-tailed Student's $t$ test in $\mathrm{g}$ and $\mathrm{i}-\mathrm{I}$. Error bars show the SDs from three independent experiments. ${ }^{*} \mathrm{P}<0.05 ; * \star \mathrm{P}<0.01$. 
a RNA pull-down for LINC00665

b

C

\section{Figure 4}

LINC00665 interacts directly with hnRNPL. a, RNA pull-down assay followed by silver staining using biotin-labeled LINC00665 sense and antisense in T24 cells. b, MS analysis of the LINC00665-enriched proteins from the RNA pull-down assay. c, d, Western blotting analysis after RNA pull-down assay with nuclear extract (c) or purified recombinant hnRNPL (d). e, Relative fluorescence of LINC00665 and hnRNPL colocalization in BCa cells. Scale bar: $5 \mu \mathrm{m}$. f, RIP assays using anti-hnRNPL for evaluating 
LINC00665 enrichment by hnRNPL. Negative control, IgG; nonspecific control, U1. g-i, RNA pull-down or RIP assays with serial deletions of LINC00665 identified the essential regions on LINC00665 required for binding hnRNPL. j, RNAalifold predicted the stem-loop structures of the hnRNPL binding sites in LINC00665. k, RIP assays after deletion of 2285-2360 nt region of LINC00665 in T24 cells. Statistical significance was assessed by the two-tailed Student's t test in $\mathrm{f}$, i, and k. Error bars show the SDs from three independent experiments. ${ }^{*} \mathrm{P}<0.05 ;{ }^{*} \mathrm{P}<0.01$.
a
b.
c
d 
LINC00665 promotes RAB27B transcription by forming a triplex structure with the RAB27B promoter. a, Heatmap of the differentially expressed genes between LINC00665-overexpressing and control BCa cells. b, qRT-PCR analysis of the change in RAB genes related to EV secretion after silencing LINC00665 in T24 cells. c, d, Western blotting analysis of RAB27B expression after LINC00665 silencing (c) or overexpression (d) in T24 cells. e, Luciferase assays with serial deletion of the RAB27B promoter in T24 cells with or without LINC00665 overexpression. f, Prediction and illustration of the triplex structure formed between LINC00665 and the RAB27B promoter. g, ChIRP analysis of LINC00665-enriched chromatin in T24 cells. h, CD spectrum of LINC00665 TFOs with RAB27B promoter TTS. Negative control, control single-stranded RNA (ssRNA) with RAB27B promoter TTS. i, FRET analysis of LINC00665 TFOs with RAB27B promoter TTS. Negative control, control ssRNA with RAB27B promoter TTS. j, k, ChIP analysis of the hnRNPL enrichment and H3K4me3 status of the RAB27B promoter in LINC00665overexpressing T24 cells with or without muting of the hnRNPL binding site. I, m, qRT-PCR analysis of RAB27B expression in LINC00665-overexpressing T24 cells (I) or 5637 cells $(\mathrm{m})$ with or without hnRNPL silencing. $n$, Representative TEM of EVs secreted by vector- or LINC00665 plasmid-transfected T24 cells with or without RAB27B silencing. Scale bar: $100 \mathrm{~nm}$. o-q, NTA (p) and quantification of EVs $(0, q)$ secreted by vector- or LINC00665 plasmid-transfected T24 cells with or without RAB27B silencing. r, S, Representative immunofluorescence images $(r)$ and quantification $(s)$ of fibroblasts treated with PKH67labelled EVs secreted by BCa cells. Scale bar: $5 \mu \mathrm{m}$. Statistical significance was assessed by 1-way ANOVA followed by Dunnett's tests in $b, g, j-m, o, q$, and $s$, or the two-tailed Student's t test in e. Error bars show the SDs from three independent experiments. ${ }^{*} \mathrm{P}<0.05 ;{ }^{*} \mathrm{P}<0.01$. 


\section{Figure 6}

LINC00665-induced EVs endow fibroblasts with the CAF phenotype by activating the TGF- $\beta$ pathway. a, Western blotting analysis showing the alteration of crucial proteins of the TGF- $\beta$ pathway in fibroblasts treated with EVs from LINC00665-overexpressing BCa cells. b, Immunofluorescence analysis of a-SMA and FAP expression in LINC00665-induced EV-incubated fibroblasts with or without SIS3 treatment. Scale bar: $5 \mu \mathrm{m}$. c, d, Flow cytometric analysis and quantification of a-SMA (c) or FAP (d) expression in 
LINC00665-induced EV-incubated fibroblasts with or without SIS3 treatment. e, Cytokine array of the CM from fibroblasts treated with EVs secreted by BCa cells transfected with vector plasmids or LINC00665 plasmids. $f$, Table showing the relative signal intensity of altered cytokines in indicated group. $g, h$, ELISA analysis of cytokine secretion by fibroblasts incubated with indicated EVs. i, Representative images and quantification of tube formation and Transwell migration of HLECs treated with fibroblast CM. Scale bars: $100 \mu \mathrm{m}$. Statistical significance was assessed by 1-way ANOVA followed by Dunnett's tests in c, d, $\mathrm{g}$, and $\mathrm{i}$, or the two-tailed Student's $t$ test in $\mathrm{f}$ and $\mathrm{h}$. Error bars show the SDs from three independent experiments. ${ }^{*} \mathrm{P}<0.05 ;{ }^{*} \mathrm{P}<0.01$. 
a

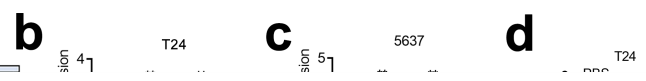

\section{Figure 7}

The EV-mediated positive loop is indispensable for LN metastasis of BCa. a, Schematic presentation of the established BCa cell and EV-induced fibroblast coculture model. b, c, qRT-PCR analysis of LINC00665 expression in $\mathrm{BCa}$ cells cultured with indicated $\mathrm{CM}$ from fibroblast with or without aHGF treatment. d, Relative transcriptional activity of LINC00665 in HGF-treated T24 cells with or without c-Myc silencing. e, Enriched motifs of c-Myc binding sites predicted by JASPAR. f, Schematic model of predicted c-Myc 
binding sequences in the LINC00665 promoter region. g, h, ChIP-qPCR analysis of c-Myc-enriched chromatin in T24 (g) and 5637 (h) cells. i, Depletion of the P1 region in the LINC00665 promoter impaired the HGF-induced LINC00665 transcriptional activity in T24 cells. j, Schematic presentation of the coculture model for analysis of the positive loop between BCa cells and fibroblasts. k, qRT-PCR analysis of LINC00665 expression in T24 cells incubated with fibroblasts. I, m, Flow cytometry analysis and percentages of a-SMA (I) and FAP (m) expression of fibroblasts. $n$, Representative images and quantification of tube formation and Transwell migration of indicated HLECs. Statistical significance was assessed by 1-way ANOVA followed by Dunnett's tests in $b-d$ and $k-n$, or the two-tailed Student's $t$ test in $\mathrm{g}-\mathrm{i}$. Error bars show the SDs from three independent experiments. ${ }^{*} \mathrm{P}<0.05 ;{ }^{*} \mathrm{P}<0.01$. 


(1)

\section{Figure 8}

Clinical relevance of the LINC00665-induced RAB27B-HGF-c-Myc positive feedback loop in patients with BCa. a, b, Representative images (a) and quantification (b) of LINC00665 expression, CAF infiltration, lymphatic vessel density, and HGF expression in BCa tissues. Scale bars: $50 \mu \mathrm{m}$. C, Correlation analysis of LINC00665 and HGF expression in BCa tissues. d, e, qRT-PCR analysis of HGF expression between BCa tissues and NATs $(n=228)(d)$ or between LN-negative $(n=175)$ and LN-positive $(n=53)$ BCa tissues $(e)$. 
$\mathrm{f}, \mathrm{g}$, Kaplan-Meier curves of the OS ( $\mathrm{f}$ ) and DFS ( $\mathrm{g}$ ) of patients with BCa with low vs. high HGF expression levels. The cutoff value is the median. $\mathrm{H}$, Representative images and quantification of tube formation and Transwell migration of HLECs treated with PBS, fibroblast CM, or CAFs. Scale bars: $100 \mu \mathrm{m} . \mathrm{i}, \mathrm{j}$, Representative bioluminescence images (i) and quantification (j) of popliteal metastatic LNs from nude mice treated with LINC00665-induced EVs with or without co-injection of aHGF $(n=12)$. Red arrows indicate footpad tumor and metastatic popliteal LN. k, Representative images and quantification of IHC staining evaluation of LYVE1-indicated lymphatic vessel density and a-SMA-indicated CAF infiltration in footpad tumors $(n=12)$. Scale bars: $50 \mu \mathrm{m}$. I, Schematic illustration of the establishment of the orthotopic xenograft model. $m$, The primary tumor size in the treatment groups $(n=12) . n$, Kaplan-Meier survival curves of the mice in the treatment group $(n=12)$. o, Proposed model of LINC00665-induced RAB27B-HGF-C-Myc positive feedback loop in promoting BCa lymphangiogenesis and LN metastasis. Statistical significance was assessed by the $\chi 2$ test in $b$, the nonparametric Mann-Whitney $U$ test in $d$ and e, or 1-way ANOVA followed by Dunnett's tests in $h, j-k$, and $m$. Error bars show the SDs from three independent experiments. ${ }^{*} \mathrm{P}<0.05 ; * * \mathrm{P}<0.01$.

\section{Supplementary Files}

This is a list of supplementary files associated with this preprint. Click to download.

- Supplementarydata.docx 